\section{Tres perspectivas sobre el desarrollo regional endógeno*}

Three perspectives on endogenous regional development

\author{
Raúl Elgueta-Rosas ${ }^{\star *}$, \\ VICENTE ESPINOZA-ESPINOZA***
}

\section{Resumen}

El artículo revisa un amplio rango de evidencia, que considera la literatura académica, informes técnicos y estudios de casos de desarrollo regional. Propone una síntesis teórica que considera tres líneas de interpretación acerca

Este artículo forma parte de la investigación Proyecto Fondecyt Regular №1120846: "Incidencia de las redes políticas en los procesos de toma de decisión para la asignación de recursos de desarrollo regional. Los Consejos Regionales en Chile 2010-2014". Parte del análisis se realizó en el marco del proyecto CONICYT/ FONDAP/15130009. Los autores agradecen la colaboración de Catalina Bustamante en la edición del texto y los comentarios de los dos evaluadores anónimos.

Instituto de Estudios Avanzados, Universidad de Santiago de Chile. Correo electrónico: raul.elgueta@usach.cl

* Instituto de Estudios Avanzados, Universidad de Santiago de Chile. Correo electrónico: vicente.espinoza@usach.cl del desarrollo regional: la primera enfatiza los problemas de funcionamiento de las instituciones; la segunda se enfoca en los mecanismos de representación política de los actores regionales, y; la tercera estudia los procesos de circulación de recursos. Estas tres líneas de interpretación intentan responder a los problemas de gobernanza y coordinación entre actores involucrados en el proceso de desarrollo regional.

Palabras clave: regionalización, descentralización, gobernanza, redes políticas.

\section{Abstract}

This paper reviews a range of evidence, which considers the academic literature, technical reports and case studies on regional development. It proposes a theoretical synthesis that considers three lines of interpretation about regional development: the first focuses on the functioning of institutions; the second focuses on the mechanisms of political representation of regional actors; and the third studies the processes of circulation of resources. These three lines of interpretation seek to address the problems of governance and coordination between actors involved in the process of regional development.

Key words: sub-national government, decentralization, governance, political networks.

\section{Introducción}

Este artículo aborda el proceso de regionalización que ha vivido Chile en los últimos veinticinco años. Para ello se revisa cómo ha 
sidoanalizadoeste proceso, paraluego proponer una agenda de estudio sobre la contribución de la institucionalidad gubernamental regional al desarrollo regional.

El proceso de regionalización chileno constituye un esfuerzo estatal que comenzó a mediados de la década del 70. El énfasis administrativo de la primera década se trasladó posteriormente hacia la voluntad de constituir órganos regionales de gobierno que actuaran como facilitadores y articuladores del desarrollo con perspectiva descentralizadora y territorial (Boisier 2003). Actualmente, Chile cuenta con 15 regiones que tienen un nivel de descentralización administrativa caracterizado como moderado. El proceso ocurre dentro de un sistema político especificado por su fuerte centralismo (Navarrete e Higueras 2014). Ello se puede observar, por ejemplo, en el hecho de que el intendente máxima autoridad regional- es designado y removido por el Presidente de la República. Ello también se observa en el hecho de que el gobierno regional no puede recaudar impuestos.

En 1992, la Ley Orgánica Constitucional $N^{\circ}$ 19.175 sobre Gobierno Interior y Administración Regional, estableció los gobiernos regionales a los cuales asignó nuevas atribuciones y recursos. El gobierno regional está compuesto por el intendente, asesorado por los secretarios regionales ministeriales que representan a los ministros, y por el consejo regional. Los consejos regionales operan en todas las regiones, manejando recursos que representaban alrededor del $25 \%$ de la inversión pública efectiva al año 2006, para descender a poco más de 20\% en 2013 (Espinoza y Bustamante 2015; Horst 2009). Hasta 2013, los consejeros que componían dicho consejo fueron elegidos indirectamente por los concejales municipales y luego, en virtud de la Ley $\mathrm{N}^{\circ} 20.678$, pasaron a ser elegidos por votación directa.

Como mencionamos, el intendente, jefe del gobierno regional, es designado o removido por el Presidente de la República en cualquier momento. También participan en el proceso de toma de decisión regional los secretarios regionales ministeriales, quienes poseen en la práctica dos "principales", pues deben responder tanto al intendente como a los ministros sectoriales del nivel central que representan. Los consejeros regionales, por su parte, son quienes aprueban o rechazan los proyectos de inversión pública regional propuestos por el intendente. A su vez, el consejo regional es dirigido por un miembro electo por los mismos consejeros regionales.

Los consejeros regionales se organizan en comisiones que revisan la pertinencia y la utilidad de los proyectos de inversión pública regional. En las comisiones se discute la incorporación a la tabla de sesiones del consejo los proyectos de inversión regional. Cabe destacar que todos los proyectos tratados en el trabajo de comisión cumplen con las condiciones mínimas técnicas para ser financiados. Salvo excepciones, los proyectos de inversión pública presentados por el intendente son aprobados en las reuniones del consejo regional. De hecho, se ha podido establecer que los proyectos han pasado por un proceso de negociación anterior a su propuesta en el consejo regional (Espinoza y Rabi 2013).

El consejo regional puede considerarse una instancia de negociación política y técnica en la cual convergen criterios distintos, tales como: políticos -regionales, locales y partidistas-, territoriales -que privilegian distintas zonas, 
principalmente provincias-, y económicos -que atienden a los intereses de algunos actores económicos-. Ello implica que los proyectos de inversión pública regional aprobados por los consejeros regionales son un instrumento mediante el cual la institucionalidad de gobierno regional pretende promover el desarrollo regional.

Desde el punto de vista metodológico, este artículo busca posicionar los distintos puntos de vista sobre el desarrollo regional basándose en la evidencia disponible. La metodología para lograrlo utiliza una revisión amplia de la literatura sobre regionalización en los últimos 25 años. A partir de dicha revisión elaboramos una síntesis para comprender las perspectivas y los desafíos de los estudios de regionalización chilenos, estableciendo una mirada que permite comprender el papel de la autoridad regional en la promoción del desarrollo regional.

El esfuerzo analítico que presenta este artículo tiene como referencia el enfoque de "evaluación basada en evidencia" (Pawson 2006; Pawson y Tilley 1997). Dicho enfoque busca reconstituir la lógica subyacente a las prácticas institucionales, utilizando como material básico estudios y análisis realizados sobre el mismo objeto. El resultado del análisis constituye una "teoría del programa" que se activa e integra dentro de relaciones sociales, vale decir, un conjunto encadenado de logros que, mediado por la interacción de los actores involucrados, conduce a un objetivo de interés.

En este artículo el objetivo de interés viene dado por el desarrollo regional endógeno, que se supone común a una serie de actores regionales, los cuales requieren desarrollar su coordinación a través de relaciones de cooperación, dado que no existe subordinación jerárquica ni mecanismos para resolver diferencias a través de terceros. El análisis que se presenta a continuación establece, entonces, las teorías de la coordinación que pueden asociarse con el desarrollo endógeno. Dado que no se trata de una evaluación, el énfasis del análisis está colocado en descubrir las debilidades del encadenamiento que se conduce desde los actores al desarrollo, para establecer una agenda de investigación.

El punto de partida teórico tiene como núcleo el enfoque de coordinación social en redes (Mascareño 2010; Lechner 1997), según el cual ni el mercado ni el gobierno por sí mismos pueden garantizar la coordinación social. La coordinación social en redes establece como condición del desarrollo regional endógeno una articulación adecuada entre el sistema político y el sistema económico, en la cual los distintos actores regionales logran niveles de colaboración, a la vez que respetan la autonomía de cada uno de estos sistemas y actores. Desde el punto de vista de las políticas públicas, la cuestión de la coordinación ha sido conceptualizada como "gobernanza", entendida como "el proceso mediante el cual los actores de una sociedad deciden sus objetivos de convivencia -fundamentales y coyunturales- y las formas de coordinarse para realizarlos: su sentido de dirección y su capacidad de dirección" (Aguilar 2009: 90).

Pueden identificarse tres perspectivas referidas a la manera en que se produce la coordinación entre actores regionales autónomos y entre distintos tipos de sistemas sociales ${ }^{1}$. La primera

Los autores que representan cada perspectiva se identifican en las secciones respectivas. 
entiende la coordinación como un problema de especificación de las funciones que deberían desempeñar los actores involucrados en el proceso de desarrollo regional. En esta línea, plantea la conducción de la política de desarrollo regional como un sistema de gobernanza multinivel -local, regional y nacional-, la cual implica, a la vez, la concertación entre actores públicos y privados.

La segunda perspectiva analiza la coordinación a partir de la representación de la ciudadanía a nivel regional. Ésta se vería facilitada por la generación de incentivos institucionales que hagan posible la relación fluida de las autoridades regionales con la ciudadanía, a través de la generación de mecanismos que propicien que los actores públicos y privados se hagan responsables frente a los otros actores.

Latercera perspectiva considera la coordinación como un proceso de circulación de recursos en ámbitos específicos ('policy network'), lo cual implica comprender el desarrollo a partir del modelo de la red social. En este caso, la coordinación se produce a través de la interdependencia de recursos, y se estudia mediante la identificación de las características estructurales que tienen los circuitos en los cuales se intercambian los recursos.

El presente artículo se inicia con el planteo del proceso de desarrollo regional endógeno en cuanto problema de coordinación y gobernanza. A continuación se desarrollan las tres perspectivas referidas a cómo resolver esta cuestión: la administrativa-funcional, la politológica derivada del principio de representación, y la reticular en la cual se estudian los procesos de circulación de recursos. Finalmente, se explica el proceso de toma de decisiones de inversión pública regional, y se propone una agenda de investigación considerando cada uno de los enfoques propuestos.

\section{El desarrollo endógeno regional: La gobernanza como un problema de coordinación gubernamental}

En la literatura especializada existe consenso al señalar que el proceso de regionalización ha sido impulsado con la finalidad de entregar protagonismo a los propios actores regionales en la implementación de su desarrollo, lo cual tendría un efecto positivo en el desarrollo nacional. Este efecto se puede sintetizar en el aforismo "el desarrollo se logrará sólo cuando las regiones en Chile estén desarrolladas", tal como lo expresó en su informe final la Comisión Asesora Presidencial en Descentralización y Desarrollo Regional:

si se quiere alcanzar un desarrollo económico y social equilibrado y sustentable, reactivar la economía potenciando los territorios, modernizar el Estado y profundizar nuestra democracia, debemos impulsar como política de Estado un conjunto de transformaciones dirigidas a una efectiva descentralización y desarrollo local-regional (2014: 10).

El planteamiento de autonomía para propiciar el desarrollo regional se aviene con el carácter subsidiario que la Constitución política reserva al Estado, lo cual le acercaría a planteamientos de desarrollo endógeno, en el sentido de que el desarrollo regional tendría origen en la propia región y no en decisiones adoptadas a nivel central $o$ en actores externos a las mismas regiones. Así, para Vázquez, este concepto "concede un papel central a las empresas, las organizaciones, las instituciones locales, así 
como a la propia sociedad civil, en los procesos de crecimiento y cambio estructural" (2004: 230). En esta dirección, la función subsidiaria del Estado no constituye garantía suficiente y, probablemente, tampoco sea necesaria para promover el desarrollo endógeno. En efecto, los actores regionales sólo promoverán el desarrollo en la medida en que ello les resulte rentable, pero no tienen la obligación de hacerlo, como tampoco de incorporar a otros actores de la realidad regional.

En un contexto en el cual el desarrollo regional resulta deseable, la finalidad de la autoridad política regional sería la promoción del desarrollo endógeno de las regiones mediante la coordinación de las dimensiones económicas, políticas y socio-culturales (Tello 2010). Sin embargo, actualmente se reconoce que la autoridad política requiere de la colaboración de una gran cantidad de actores que no dependen de ella, y que no comparten, necesariamente, las mismas finalidades. La literatura de administración pública caracteriza esta situación como un problema de gobernanza (Aguilar 2009). Dicho problema implica reconocer las dificultades del ejercicio de las funciones de gobierno en el contexto de sociedades complejas. De manera más general, las dificultades para impulsar el desarrollo endógeno pueden ser conceptualizadas también como problemas de coordinación entre los sistemas político, económico y social.

A mediados de la década de los 90, Lechner (1997) señalaba la existencia de tres principios de coordinación: económico, político y reticular. La coordinación económica supone que el desarrollo se articula a través del sistema de precios establecidos en los intercambios de libre mercado, lo cual establece una coordinación descentralizada, sin jerarquías. Los mecanismos de coordinación política, por contraste, son centralizados y jerárquicos, jugando un papel central la autoridad de gobierno. Finalmente, la coordinación reticular se refiere a la dependencia recíproca que da origen a una red entre los distintos actores, vale decir, una pauta subyacente a sus interacciones. La coordinación en red involucra un incremento en los niveles de complejidad social, pues "supone la interrelación de distintos actores y sistemas con marcada autonomía para definir intereses y procedimientos de operación propios" (Mascareño 2010: 111). En un sentido similar, De la Maza, Cunill y Joignant (2012) apuntan a la existencia de estas tres formas de regulación social en el desarrollo territorial: mercado, autoridad/jerarquía y redes.

Las cuestiones de gobernanza y coordinación aluden a un mismo fenómeno, observado desde diferentes puntos de vista. Si se analiza desde la perspectiva de la autoridad gubernamental, el desarrollo regional endógeno constituye un problema de gobernanza, ya que para promover este tipo de desarrollo la autoridad política requiere de la colaboración de actores que no dependen jerárquicamente del gobierno en cuanto a recursos, intereses o reglas de funcionamiento. Así lo señala un estudioso del tema: "[e]l actuar del gobierno no está en condiciones de dirigir a la sociedad a menos que conjunte y coordine sus recursos directivos y mecanismos de coordinación con los recursos y mecanismos directivos de otras instancias sociales, el mercado y la sociedad civil organizada" (Aguilar 2009: 86).

Mirado desde el punto de vista societal, el desarrollo es consecuencia de una adecuada articulación entre los sistemas político, 
económico y social. Tanto el concepto de coordinación como el de gobernanza identifican la necesidad de colaboración entre actores que no comparten finalidades, pero sí los recursos necesarios para el logro de un objetivo de interés común. En dicha colaboración, si bien el gobierno no cuenta con todos los recursos para imponer un determinado modelo de desarrollo, normalmente asume la responsabilidad de liderar dicho proceso.

En conclusión, tanto el concepto de gobernanza como el de coordinación se basan en el reconocimiento de las dificultades que tiene el gobierno, en tanto expresión del sistema político, para conducir el desarrollo. Si bien los actores políticos cuentan con legitimidad para establecer las prioridades del desarrollo, no cuentan con los instrumentos y recursos suficientes para llevarlo a cabo por sí mismos. Además, aunque el desarrollo es consecuencia de los intercambios económicos, políticos y culturales existentes en las regiones, estos intercambios no resuelven la cuestión de las finalidades o del tipo de desarrollo que éstas debieran tener. Es por dicha coincidencia entre la coordinación y la gobernanza que hablamos del problema de coordinación gubernamental. Dicha coordinación releva la centralidad de la función gubernamental aunque se reconocen las limitaciones con que cuentan los gobiernos regionales para liderar los procesos de desarrollo regional.

\section{Perspectivas de coordinación gubernamental regional en Chile}

La coordinación gubernamental reconoce que el desarrollo regional endógeno es consecuencia de una adecuada articulación entre actores gubernamentales, actores privados y actores de la sociedad civil regional (De la Maza, Cunill y Joignant 2012). ¿Cómo el gobierno regional puede promover el desarrollo regional endógeno con la participación de actores diversos? La respuesta supone cumplir con el requisito de colaboración de los diferentes actores que inciden en el desarrollo regional, respetando la autonomía de funcionamiento de los actores y su independencia para fijar sus propias finalidades.

Mascareño (2010) identifica tres mecanismos de coordinación que se han propuesto en la generación depolíticas públicas durantelaúltima década. En primer lugar, las "policy networks" establecen coordinaciones basadas en la dependencia mutua de recursos, expresadas en mecanismos como las licitaciones de obras públicas bajo el sistema de concesiones. En segundo lugar, la orientación "contextual" establece coordinaciones basadas en la legitimidad procedimental de mecanismos que facilitan la elaboración de acuerdos razonables, cuyo modelo general son las mesas de deliberación que se manifiestan bajo formas como las comisiones presidenciales o las mesas de diálogo. En tercer lugar, se encuentra el modelo de derecho reflexivo o "política de opciones" en el cual la coordinación apela a una tercera parte que no posee intereses en el proceso, como ocurre con los mecanismos de arbitraje.

Para Mascareño (2010), estas tres formas suponen coherencia en la interrelación de sistemas y actores autónomos, derivada de la orientación en tareas específicas. Además, quienes participan del proceso desarrollan una visión común y promueven un nivel tolerable de autolimitación de la autonomía (Jabko 2004). Los mecanismos son procedimentales y aspiran 
a elevar la reflexividad de sistema y actores por medio de la atención a las consecuencias de sus operaciones autónomas.

Si bien los mecanismos de coordinación propuestos por Mascareño (2010) permiten responder a los problemas de coordinación en un nivel general, no resultan pertinentes para dar cuenta de la manera en que los gobiernos regionales intentan promover el desarrollo regional. El carácter procedimental de los mecanismos propuestos involucra que la cooperación se convierte en un fin en sí misma y que la convergencia de finalidades termina con el procedimiento, sin estabilizar, necesariamente, mecanismos de cooperación entre los actores involucrados. Desde el punto de vista procedimental, los mecanismos tienden a ser contingentes o temporales y no permanentes. El acople entre procedimientos y la construcción de instituciones está mediado por el involucramiento de los actores en la construcción de las alternativas, lo cual puede, incluso, llevar a que modifiquen sus posiciones iniciales (Jabko 2004).

Es por ello que en este artículo se conceptualizan mecanismos de coordinación más estables y que institucionalicen la colaboración entre los actores que participan del desarrollo regional endógeno. Para esto se proponen tres perspectivas referidas a la manera en que se resuelven los problemas de coordinación gubernamental. Todas estas perspectivas están presentes en los estudios sobre regionalización en Chile. Aunque proceden de enfoques disciplinarios disímiles, todas ellas respetan la autonomía de los actores involucrados en el desarrollo regional, y especifican mecanismos que propician una coordinación permanente en la promoción del desarrollo endógeno.

\subsection{La perspectiva administrativa-funcional}

Desde el punto de vista administrativofuncional, el principal problema de coordinación gubernamental lo constituye la poca claridad en la especificación de las funciones y las competencias de los actores que deben coordinarse. Por tanto, la adecuada coordinación gubernamental se lograría mediante la especificación clara de las funciones y las tareas de los distintos actores que participan en el desarrollo regional.

Los problemas de delimitación de las funciones serían consecuencia de las dificultades propias del proceso de descentralización, esto es, la transferencia de poder político, fiscal y administrativo desde el nivel central a los niveles sub-nacionales de gobierno -regional y municipal-. Buenapartedelosanálisisinspirados en esta perspectiva buscan establecer qué procesos corresponden efectivamente a la descentralización, por contraste con la desconcentración administrativa, la delegación de funciones o la funcionalidad territorial, para establecer sus consecuencias económicas, políticas y administrativas (Mardones 2008; Boisier 2004).

El proceso de transferencia de funciones a niveles sub-nacionales ha incrementado la cantidad de actores gubernamentales que deberían colaborar en la promoción del desarrollo regional, lo cual ha implicado mayores dificultades de coordinación para los actores públicos nacionales, regionales y locales. Estas dificultades han sido presentadas en el marco de las complejidades que plantea la 'gobernanza multinivel' (Horst, Irarrázaval y Pérez 2009; Montecinos 2006). Este concepto fue originalmente aplicado al proceso de 
integración de los estados miembros de la Comunidad Económica Europea, y apunta a la necesidad de generar una función de gobierno entre actores de nivel nacional, regional y local, así como entre el ámbito gubernamental y no gubernamental (Bache y Flinders 2004).

En la literatura sobre descentralización en Chile se han identificado una serie de dificultades para establecer una adecuada 'gobernanza multinivel', que incluye tanto a actores del nivel central como a actores del nivel regional. Serrano (2001) indica como problema central la confusión en las atribuciones específicas del gobierno regional y del consejo regional. Recientemente, el equipo del Programa Ciudadanía y Gestión Pública de la Universidad de Los Lagos ha presentado un informe sobre Articulación en el Proceso de Descentralización en tres regiones chilenas, cuyos resultados también resaltan estos problemas para una adecuada 'gobernanza multinivel', así como la confusión y la disputa en las atribuciones de los organismos de gobierno (Aroca y Atienza 2010; De la Maza 2010; González 2010; Navarrete 2010). Esta perspectiva se ha plasmado también en el Informe de la Comisión Asesora Presidencial para la Descentralización y el Desarrollo Regional:

Una definición constitucional precisa de las competencias de los gobiernos regionales no sólo ayuda a reducir los eventuales y previsibles conflictos de competencias que puedan suscitarse entre los distintos niveles de gobierno, sino que es, además, condición necesaria para atribuir al Tribunal Constitucional la función esencial de dirimir tales controversias (2014: 11).

Un segundo tipo de dificultades de 'gobernanza multinivel' importante en el caso chileno se manifiesta en el vínculo que establecen los gobiernos regionales con los actores de diferente nivel administrativo. Existen evaluaciones críticas sobre la situación de los gobiernos regionales, que incluyen el incremento en el control centralizado sobre el uso de recursos de inversión pública regional, a través de "provisiones" fijadas por el nivel central (Garcés y Valdés 2009; Horst 2009, 2008; Larraín 2008), las cuales restringen el uso de fondos que deberían ser orientados considerando las necesidades de las regiones.

Estas dificultades entre diferentes niveles administrativos han sido estudiadas especialmente en la relación que establece el nivel local -municipal- con el nivel regional, donde los actores municipales buscan incidir en la toma decisiones a nivel regional para obtener recursos de inversión pública (Espinoza 2006). En la misma línea, Nickson (2011) señala que el proceso de descentralización chileno sería un caso excepcional en América Latina, que se correspondería con un estilo "gerencial" derivado de los traspasos de funciones del nivel central al ámbito municipal. Otra parte de la literatura destaca que el proceso de descentralización chileno ha focalizado la entrega de recursos en el ámbito municipal antes que en el regional, produciendo problemas de gobernanza regional. En efecto, las localidades cuentan con escasa incidencia en la definición de las metas de desarrollo regional, carecen de capacidad técnica para implementar proyectos complejos, lo cual se suple con ajustes coyunturales (Espinoza y Bustamante 2015; Espinoza y Rabi 2013).

Mardones (2006) sostiene que la principal consecuencia del proceso de descentralización ha sido la concentración de poder en la figura del alcalde. Efectivamente, a nivel comunal se encuentra la operación de un modelo 
"gerencialista" de descentralización, cuya única forma de escape de los problemas de funcionamiento residiría en la voluntad política del alcalde para impulsar la participación ciudadana (Montecinos 2006). La relevancia que alcanza el nivel local puede tener efectos positivos, pero también tiene un lado oscuro. Una perspectiva optimista destaca que el poder local puede ser una alternativa frente al poder de las corporaciones trasnacionales, las cuales se apoyan en el gobierno central para insertarse en los países (Fløysand, Barton y Román 2010). Sin embargo, en su lado menos brillante, el poder local se podría ver asociado al clientelismo, la corrupción o la captura por los intereses de la elite local (Durston 2012; Montecinos 2011; Horst, Irarrázaval y Pérez 2009; Barozet 2006, 2005a, 2005b; Durston et al. 2005; Rehren 2002).

Un tercer tipo de problema que destaca en la perspectiva administrativa-funcional corresponde a las asimetrías en los niveles de competitividad regional. La competitividad constituye una condición necesaria del desarrollo regional, la cual se apoya en una combinación de competencia y cooperación entre actores económicos, destinadas a formar agrupamientos productivos ${ }^{2}$. En este aspecto, el rol del estado consiste en proveer bienes públicos, tales como infraestructura, recursos humanos, información, conocimiento científico y adelantos tecnológicos, mientras que los actores privados se encargan de aprovechar sus ventajas comparativas para impulsar el desarrollo (Felzenstein y Olavarría 2011; PérezAlemán 2005; Silva 2005; Bitrán 2004; Montero 2004; Montero y Morris 1999; Porter 1998).

Existen políticas públicas destinadas a impulsar "clusters productivos" (Rivas 2012).
En este marco, una adecuada coordinación entre actores públicos y privados, así como entre los actores gubernamentales de nivel municipal, regional y nacional facilitaría el desarrollo regional. La coordinación entre actores se logra a través de la definición de una estrategia, esto es, un plan de acción común que apunta a un objetivo concreto, el cual se traduce en la elaboración de instrumentos de planificación pública que incluyen a los diversos participantes. La planificación es liderada por el gobierno regional mediante un plan de desarrollo regional concertado en conjunto con los distintos actores involucrados de la región. En definitiva, el desarrollo regional se lograría mediante un gran acuerdo regional que especifique las competencias de los distintos actores regionales, y no solamente de los gubernamentales. La experiencia de las "Estrategias de Desarrollo Regional" que expresan esta perspectiva administrativa de coordinación muestra que ellas no logran movilizar a los actores en acciones de desarrollo significativas (Rivas 2012; Centro de Estudios en Economía y Negocios 2011).

En síntesis, la perspectiva administrativafuncional permite identificar tres tipos de dificultades para una correcta coordinación a nivel regional. La primera se asocia con la especificación de competencias de las autoridades gubernamentales en las diferentes reparticiones gubernamentales de la institucionalidad pública regional. La segunda destaca la disputa entre el nivel regional respecto del ámbito local. La tercera se relaciona con una inadecuada planificación regional que propicie la competitividad de las regiones.

La solución para resolver estos tres tipos de dificultades consistiría en la definición clara y 
compartida de las competencias de los actores que inciden en el desarrollo regional. Esta definición se lograría principalmente mediante una planificación regional que establezca de manera clara y consensual cuáles son las funciones que deben desempeñar las autoridades regionales, de manera de coordinarse con los actores privados así como con los otros actores públicos.

\subsection{La perspectiva política de la coordinación: La representación}

La perspectiva de coordinación, en tanto representación, identifica como principal dificultad para el desarrollo regional la débil institucionalidad regional, expresada en la limitada responsabilidad que los actores gubernamentales asumen por su comportamiento frente a los otros actores. Los problemas de coordinación gubernamental tienen su origen en el escaso desarrollo de sistemas de control y balance regional y, en particular, las dificultades para la conformación de mecanismos de representación que propicien la existencia de una opinión pública regional.

A diferencia de la perspectiva administrativafuncional, en el caso de la representación, los sistemas político-económicos y de actores sociales se coordinan a través de la necesidad de responder a requerimientos de los otros sistemas, y no mediante una adecuada especificación de las funciones de los actores que deben coordinarse. Asimismo, los conflictos entre actores se resuelven mediante los mecanismos institucionales que generan las condiciones para que los sistemas respondan a otros sistemas (Avendaño 2008).
Dentro de los significados que puede tener la representación, tomaremos aquél que se refiere a la responsabilidad u obligación de responder, es decir, ante quién es responsable la autoridad. Sartori (2002) caracteriza de este modo la representación electoral: "[l]a teoría electoral de la representación es, en efecto, la teoría de la representación responsable: su problema no es el de satisfacer el requisito de la semejanza, sino de asegurar la obligación de responder" (2002: 266).

El marco legal regional se ha comenzado a modificar con la finalidad de introducir mecanismos más democráticos en la elección de las autoridades del ejecutivo regional, lo cual involucra que las autoridades regionales responden ante la ciudadanía ${ }^{3}$. Al respecto, el primer paso dado fue la elección directa de los consejeros regionales en las elecciones del año 2013. A ello se debe agregar la propuesta de elegir, en el futuro, al intendente por votación popular, presente ya en varios anuncios e incluida como segunda medida fundamental por la Comisión Asesora Presidencial para la Descentralización y el Desarrollo Regional (2014). Sin embargo, la representatividad del nivel nacional-gubernamental permanece en los cargos de secretarios regionales ministeriales, que son los representantes de los ministerios a nivel regional, y cuyo mandato depende de la confianza del ministro.

En un sentido similar de responsabilidad en los actos públicos, se ha elaborado el concepto de 'accountability' que enfatiza la rendición de cuentas y la transparencia. Los análisis de 'accountability' distinguen un sentido horizontal

Hasta 2013 los consejeros regionales fueron elegidos indirectamente por los concejales comunales de cada región agrupados por provincias. 
y otro vertical en la rendición de cuentas (O’Donnell 1999). La horizontal se realiza frente a otras instituciones gubernamentales y puede implicar el balance de poder, ya que permite la generación de mecanismos de control recíproco entre actores gubernamentales (Peruzzotti y Smulovitz 2002). En cambio, la vertical se realiza frente a la ciudadanía, principalmente en actos electorales -'electoral accountability'- y, en algunos casos, cuando la ciudadanía protesta y realiza movilizaciones sociales para exigir una rendición de cuentas de sus autoridades -'social accountability'- (Peruzzotti y Smulovitz 2002; Smulovitz y Peruzzotti 2000).

La 'accountability' se ha utilizado tanto en los ámbitos públicos como privados, y puede ser aplicada a la relación entre ambos. Esto es posible ya que se supone que la vinculación entre actores es un proceso de comunicación, en el cual quienes se coordinan deben responder y justificar sus comportamientos frente a otros actores. Es por ello que la responsabilidad se puede asociar con el concepto de opinión pública regional, espacio en el cual los actores deben justificar razonablemente su comportamiento frente a la ciudadanía.

Desde la perspectiva de la representación, se entiende que tanto la calidad del desarrollo como la calidad de la democracia son consecuencia de una adecuada articulación de los distintos tipos de actores que deben justificar razonablemente sus acciones. Sin embargo, en ocasiones, los mecanismos de 'vertical accountability' -electoral y socialpueden servir para reforzar las tendencias centralistas (De la Maza 2012). Además, estos mecanismos verticales podrían reforzar la desigualdad, ya que tenderán a participar electoralmente y a movilizarse aquellos actores más competentes de estratos más altos (Smulovitz 2002).

El diseño institucional regional comporta la necesidad de elaborar un marco institucional en el cual las instituciones regionales estén obligadas a responder a la ciudadanía. Esto implica la generación de 'electoral accountability' a nivel regional que facilite la comunicación del sistema político a nivel regional con la sociedad civil y el sistema económico del mismo nivel, para formar mecanismos virtuosos que faciliten el desarrollo regional endógeno. Estos mecanismos han sido planteados por la Comisión Asesora Presidencial para la Descentralización y el Desarrollo Regional (2014) tanto en la novena medida esencial Democracia Local y Regional-, en la que se propone limitar la reelección de autoridades regionales, como en la décima medida Fortalecer la Participación Ciudadana a través de la incorporación de Plebiscitos Regionales-. Dichas medidas indican carencias en los mecanismos de representación regional. Así lo sostiene una experta en la materia ${ }^{4}$ :

\section{Los votantes no pueden pronunciarse sobre la política regional, que no es discutida en campaña electoral alguna ni tampoco sobre las autoridades regionales ya que las elecciones municipales solamente versan sobre los problemas del municipio y nunca sobre los de la región (Rufián 2009: 95).}

En síntesis, la perspectiva de la representación permite identificar dos tipos de problemáticas de coordinación. La primera enfatiza la carencia de mecanismos regionales de responsabilización derivados de la falta de

Dicha expresión se formuló con anterioridad a la reforma que implicó la elección directa de consejeros regionales el año 2013, aunque la afirmación respecto al contenido de las campañas requiere de un estudio particular, en la medida en que no está garantizado por la elección directa de los representantes. 
elección directa de la principal autoridad del poder ejecutivo de la región, y de mecanismos adicionales de balance del poder regional. El segundo tipo de problemas se relaciona con la dificultad de conformar una opinión pública regional, el que se deriva también de la falta de elección directa del intendente de la región.

\subsection{La gobernanza en red: La centralidad de los patrones de circulación de recursos}

La tercera perspectiva comprende el desarrollo regional endógeno como un proceso de 'circulación de recursos', en el cual se produce una necesaria 'coordinación informal' entre actores del sistema de poder regional. Los recursos que circulan son económicos y materiales, por ejemplo, financiamientos, créditos o infraestructura; recursos políticos como poder e influencia; recursos simbólicos como prestigio, información o conocimiento especializado. Cada recurso está asociado con actores específicos, generando una situación de dependencia mutua en la medida en que ellos sean necesarios para el desarrollo regional. Las modalidades mediante las cuales circulan los recursos pueden favorecer el desarrollo endógeno siempre que los recursos alcancen toda la red, posibilitando su acceso a los actores que cuentan con menos recursos. En este sentido, el gobierno regional cumple con la función de potenciar la conformación de alianzas estratégicas que propicien un intercambio de recursos, beneficiando de este modo el desarrollo estratégico.

La circulación de recursos puede ser estudiada como una 'policy network' (Klijn 1998). En este tipo de red los participantes poseen en común el interés en los resultados de las decisiones, lo cual define el campo de actores de la política pública -‘policy domain’- (Knoke 1993; Knoke, Laumann y Kim 1985). A pesar de su coordinación, estos actores no poseen relación de jerarquía entre sí, ni tampoco reconocen una autoridad común que pueda resolver sus disputas de interés, por lo que cabe entenderlos como una organización en red (Klijn 1998; Podolny y Page 1998; Knoke y Pappi 1991). Esta organización implica procesos de "gobernanza en red" que incentivan el desarrollo de formas de coordinación horizontal orientadas a la cooperación, las cuales dan origen a redes de política pública-'policy networks'- entre actores que reconocen su dependencia recíproca en relación con los recursos que posee cada uno.

Un primer tipo de problema que se destaca a partir de esta perspectiva radica en que una posible concentración inicial de los recursos se convierta en una distribución desigual de los mismos. En esta dirección, la circulación de recursos puede clausurarse a algunos actores, limitando la posibilidad de generar emprendimientos e innovaciones favorables al desarrollo regional. Esta preocupación está presente en el Informe de la Comisión Asesora Presidencial para la Descentralización y el Desarrollo Regional (2014), que tiene como objetivo combatir la desigualdad territorial. El proceso de generación de confianzas en participantes de la red que puedan derivar en 'joint ventures' o alianzas estratégicas requiere de un cuidadoso monitoreo de las interacciones, de forma que los actores pasan de desconocidos a conocidos (Zeng y Chen 2003; Kollock 1998).

Un segundo tipo de problema que se deriva de esta perspectiva reconoce que la 'gobernanza en red' puede estar asociada con prácticas de exclusión, tráfico de influencia y, más aún, corrupción (Porras y Pino 2010; Mardones 
2008; Montecinos 2006; Rehren 2002). Dicho problema ha sido estudiado mediante el concepto de clientelismo, con el cual se analiza el intercambio de recursos económicos del gobierno a cambio de apoyo político.

Arturo Valenzuela (1977) realizó hace casi cuatro décadas el estudio clásico sobre extracción de recursos públicos para iniciativas locales, que caracteriza la política local como un espacio de 'brokers"s. Valenzuela (1977) identificó dos mecanismos complementarios de intermediación para obtener recursos públicos. El primero era un 'broker' local, usualmente un regidor o alcalde, que podía traspasar su propio apoyo electoral a los partidos nacionales. El segundo mecanismo incluía un 'broker' nacional, por lo general un parlamentario, que podía "sacar" recursos del nivel central, como ayuda directa a clientelas locales, utilizando su prestigio para facilitar el acceso a funcionarios de alto nivel, o por medio de la modificación del presupuesto nacional para lograr impacto a nivel local.

El intercambio que realizaban ambos tipos de 'brokers' presentaba la particularidad de que era semi-competitivo, ya que sus clientelas podían seleccionar entre 'brokers' diferentes para obtener acceso a recursos públicos, tal como los partidos también podían elegir o cambiar entre distintos 'brokers'. Los recursos públicos para las localidades provenían de modificaciones al presupuesto nacional, derivadas de complejas negociaciones realizadas en el parlamento nacional. Mientras las localidades recibían los recursos, alcaldes y regidores se encargaban

Mantenemos la expresión en inglés dado que no existe un equivalente para la intermediación en el ámbito político, como sí lo hay en el uso comercial: corredor de bolsa o corredor de propiedades. de garantizar el apoyo electoral a los diputados. De esta manera, el modelo de intermediación tipo ‘broker' contribuyó a que la lógica electoral local fuera complementaria con la lógica electoral nacional.

En la actualidad hay restricciones constitucionales que impiden hacer uso del mecanismo de modificación del presupuesto, pues los parlamentarios no pueden presentar iniciativas legales que involucren recursos públicos. Tampoco pueden solicitar aumentos en el presupuesto nacional, que son de iniciativa exclusiva del Presidente de la República, ya que en la discusión presupuestaria no pueden incrementar ni tampoco introducir modificaciones o nuevas partidas en dicho presupuesto.

Sin embargo, según recientes investigaciones, los fondos de inversión pública regional que son aprobados por el consejo regional constituyen alrededor de $20 \%$ del presupuesto nacional de inversión (Espinoza y Rabi 2013). Adicionalmente, Durston (2012) da cuenta de la persistencia de modelos de relaciones clientelares en la forma de implementación actual de las políticas públicas. De modo semejante al modelo de brokers, el semiclientelismo caracterizado por Durston (2012) propicia el pluralismo o condiciones semicompetitivas de elección de autoridades.

En síntesis, se pueden identificar dos tipos de dificultades para una correcta coordinación a nivel regional que resaltan a partir de la perspectiva de circulación de recursos. El primer tipo de dificultad se deriva de la asimetría en la distribución de recursos con que cuenta la red de poder regional. El segundo tipo de dificultad se vincula con el intercambio de 
recursos económicos públicos a cambio de apoyos políticos, o los problemas asociados al clientelismo y formas afines de relación entre autoridades y votantes.

\section{Desafíos y agenda de investigación para el desarrollo regional}

Las secciones siguientes recapitulan las principales insuficiencias detectadas en el análisis de las perspectivas sobre coordinación, para plantearlas en términos de líneas de investigación asociadas con el desarrollo regional. Tanto la detección de problemas como la identificación de líneas de investigación tienen como foco los problemas de coordinación entre actores $\mathrm{y}$, de ninguna manera, aspira a incorporar todos los problemas asociados con el desarrollo regional.

\subsection{Desafíos y agenda en la perspectiva funcional-administrativa}

Esta perspectiva de la coordinación otorga centralidad a las competencias funcionales que tienen los distintos actores involucrados en el proceso de toma de decisiones de inversión pública regional. Un primer problema de coordinación gubernamental que se identificó fue la falta de especificidad en las funciones que desempeñan los actores públicos que intervienen en el desarrollo. Esta problemática ha sido estudiada analizando la eficiencia del proceso de toma de decisiones de inversión pública regional. Buena parte de la literatura enfatiza los riesgos derivados de una insuficiente especificación de las funciones del gobierno regional. Esta insuficiencia redundaría, por ejemplo, en duplicación en las funciones respecto del consejo regional, en presiones de los ministerios a nivel central, o incluso en exigencias de las autoridades del nivel local.

Un aspecto clave para la agenda de investigación consiste en estudiar la complementariedad y/o la competencia entre el consejo regional y el gobierno regional en el proceso de toma de decisiones de inversión pública regional. Puesto que las características de la colaboración entre el consejo regional $y$ el gobierno regional no se encuentran especificadas legalmente, se pueden analizar las diferentes modalidades de organización que adoptan los procesos de toma de decisiones de inversión regional. Si bien la coordinación se vuelve más improbable cuando no existe una adecuada especificación de las competencias, ello genera un espacio para la innovación y el aprendizaje de los actores regionales. Por tanto, se pueden estudiar modalidades informales de coordinación y de resolución de controversias entre agentes gubernamentales. Ello implicará resultados diferenciados entre regiones, y aprendizajes expresados en innovaciones que pueden generalizarse a distintas regiones.

Un segundo tipo de problema que se identificó en esta perspectiva son las dificultades que tienen los gobiernos regionales para coordinarse con actores gubernamentales de diferente nivel administrativo, en particular, referido a los gobiernos locales-municipales. Mardones (2001) sintetizó la situación del régimen interior como correspondiente en su esencia a un Estado unitario desconcentrado, una situación que se mantiene por 14 años. El fundamento de este juicio se encuentra en que los gobiernos regionales no poseen autonomía política o legislativa ni generan ingresos propios, mientras que las municipalidades tampoco 
cuentan con atribuciones para administrar los recursos que les genera la aplicación de impuestos y derechos.

Esta crítica no cuestiona, sin embargo, el papel que juega el gobierno regional en la asignación de los recursos disponibles que se multiplicó nueve veces entre 1989 y 2005 (Marcel 2006). La Comisión Asesora Presidencial para la Descentralización y el Desarrollo Regional (2014) ha propuesto en su cuarta medida esencial la creación de un Sistema de Administración de Áreas Metropolitanas, lo cual da cuenta de algunas iniciativas que intentan resolver los problemas de coordinación multinivel. Desde el punto de vista de la investigación, ello implica estudiar la manera en la cual se dirimen los conflictos entre actores de diferente nivel en todas las regiones, así como revisar la operación de las coordinaciones más exitosas.

Un tercer problema de coordinación identificado en esta perspectiva corresponde a la asimetría entre los niveles de competitividad regional. Ello puede ser estudiado haciendo una comparación sistemática de la forma en que se coordinan entre regiones los actores con altos niveles de competitividad y con bajos niveles de competitividad. Se podría estudiar la articulación entre la inversión pública regional y el desarrollo productivo regional, centrándose en ámbitos productivos considerados estratégicos por los instrumentos de planificación regional.

\subsection{Desafíos y agenda en la perspectiva de la representación}

La principal dificultad identificada dentro de esta perspectiva fue la carencia de mecanismos regionales de responsabilización, lo cual deriva de la falta de mecanismos de representación a nivel regional. El gobierno regional mezcla autoridades cuyos mandatos provienen de distintos procesos, de forma de mantener un vínculo de lealtad entre la autoridad y quien la elige o selecciona, lo que puede plantear conflictos entre actores. Los consejeros regionales tienen voz y voto en el financiamiento de los proyectos de inversión pública regional. Éstos son elegidos por ciudadanos que pertenecen, por lo general, a provincias que agrupan varios municipios.

Es razonable suponer que los incentivos establecidos por el sistema de elección de los consejeros les llevarían a privilegiar los proyectos de las provincias que le eligieron, por sobre los que potencian el desarrollo regional. En esta línea, los consejeros regionales podrían entrar en conflicto con otros actores regionales tales como el intendente designado por el Presidente de la República-, y con los secretarios regionales ministeriales dependientes de la intendencia y los ministros respectivos-. Teóricamente, los consejeros regionales también podrían entrar en conflicto con alcaldes o parlamentarios, cuyo mandato proviene de otro proceso eleccionario.

Una agenda de investigación que estudie los conflictos derivados del origen del mandato y su impacto en el desarrollo regional analizaría si los mecanismos de selección de las autoridades implican la generación de vínculos de lealtades frente a aquellos que los han elegido. Para ello se hace necesario estudiar en profundidad los procesos electorales del consejo regional. Así, se puede dilucidar si los consejeros regionales representan a la región como indica la ley, o si representan a las provincias, o a los partidos políticos y sus clientelas (Valenzuela 1977). 
También se puede revisar la existencia de otros mecanismos de rendición de cuentas -'accountability'- a nivel regional, por ejemplo, los mecanismos de 'accountability' horizontal, - los que emanan de los instrumentos de planificación regional en procesos de autoevaluación de la gestión del gobierno regional. Debe recordarse que incluso pueden emerger mecanismos de rendición de cuentas derivados de escándalos que impliquen el surgimiento de mecanismos de 'social accountability', por lo que se pueden estudiar las dinámicas y los repertorios que concurren en aquellas movilizaciones de actores que exigen la rendición de cuentas de actores regionales.

El segundo tipo de dificultad que se ha señalado proviene de los inconvenientes en la conformación de una opinión pública regional. Ello puede ser estudiado de dos maneras. La primera consiste en estudiar las campañas de los consejeros regionales, y determinar si su agenda se estructura en torno a las problemáticas regionales 0 , en cambio, responden a temáticas municipales $\mathrm{u}$ otro tipo de agendas. La segunda dice relación con realizar un seguimiento de la agenda pública de los consejeros regionales en los medios de comunicación regionales, y examinar si ocupan un papel importante en ellos. La concurrencia con las elecciones presidenciales, de diputados y senadores, como sucedió en 2013, podría dificultar mucho la importancia del cargo de consejero regional, así como las características particulares de las candidaturas. La pregunta clave a responder es de qué modo las elecciones regionales contribuyen a dar mayor visibilidad y a reforzar el debate, conformando una opinión pública regional.

\subsection{Desafíos y agenda en la perspectiva de la circulación de recursos}

En el enfoque de circulación de recursos la primera dificultad que se distinguió reside en la asimetría en la distribución de los recursos con que cuenta la red de poder regional. Desde el punto de vista de la investigación, para esclarecer esta dificultad se pueden generar las redes que acompañan el proceso de toma de decisiones de inversión pública regional, tomando como guía la circulación de recursos tales como la información. En primer lugar, ello se puede realizar identificando la centralidad de los actores en la red de consulta sobre las decisiones a las que recurren los consejeros regionales: si existen algunos actores regionales más consultados, ellos ocupan posiciones centrales en la red de toma de decisiones. Una segunda estrategia de investigación consiste en realizar un análisis documental de los principales proyectos o áreas temáticas, de tal forma de reconocer una red de política pública regional ('policy domain'), y analizar las relaciones entre los actores involucrados.

El segundo tipo de dificultad que se encontró se relaciona con la existencia de redes clientelares, en las cuales se intercambian recursos económicos públicos a cambio de apoyos políticos. Estos circuitos de intercambio pueden ser identificados a través de un análisis etnográfico de los consejeros regionales, tanto en periodo de elecciones como durante su gestión, de manera que sea posible distinguir la distribución de recursos, por un lado, y el correlato en los resultados electorales, por el otro. Una segunda modalidad de estudio consiste en analizar la participación de los actores involucrados -'stakeholders'- en la aprobación de proyectos de inversión pública 
regional, cuyos principales destinatarios son las municipalidades.

De forma complementaria se puede estudiar la conformación de elencos de candidaturas electorales entre los distintos tipos de elección municipal-concejalesyalcaldes-, parlamentarias -diputados y senadores-, y de consejeros regionales. El argumento del 'broker' señala las dificultades que ellos experimentan al cambiar de interlocutor en los tipos de intermediación en que se especializan. La presencia de relaciones clientelares llevaría a esperar un escenario de poca movilidad entre los cargos de elite de las autoridades electas a nivel regional.

\section{Conclusiones}

Las transformaciones que ha vivido el país en los últimos treinta años han acarreado un cambio en la manera en la cual el gobierno propicia el desarrollo regional, dado que el gobierno regional requiere de la colaboración de otros actores autónomos en su funcionamiento y finalidades. En este artículo se ha señalado que esta situación es un problema de coordinación gubernamental, concepto que reconoce el problemadecoordinación entreactores públicos y privados, destacando que ello no implica la renuncia de la responsabilidad del gobierno regional en la promoción del desarrollo. Esto involucra reconocer la necesidad de estudiar los mecanismos mediante los cuales el gobierno podría favorecer la colaboración entre actores -públicos y privados- con funcionamientos e intereses autónomos.

A partir del análisis de estudios sobre regionalización en Chile, se han planteado tres posibles modos de enfrentar el problema de la coordinación gubernamental. El primero enfatiza la competencia funcionaladministrativa, perspectiva según la cual el desarrollo y la coordinación se producirían mediante una adecuada especificación de las funciones que desempeñan los diversos actores y sistemas, y, a su vez, supone establecer mecanismos que especifiquen finalidades que vuelvan competentes a los actores. Estos mecanismos se asocian con una agenda de investigación de los procesos de inversión pública regional preocupada por el correcto funcionamiento del sistema político regional, con la especificación de las competencias de los actores gubernamentales, así como con la formulación de instrumentos de planificación que involucran a actores públicos y privados en la tarea de implementar el desarrollo regional.

Otro mecanismo de coordinación se centra en la representatividad. Este mecanismo implica la generación de incentivos que facilitan la responsabilización de los actores implicados en una opinión pública regional. Bajo esta concepción, las "descoordinaciones" se producen debido a que no existen incentivos para que los actores involucrados en el desarrollo regional se hagan responsables frente a la ciudadanía regional o frente a otras instancias regionales. Según la literatura revisada, la introducción de mecanismos de elección directa de autoridades del poder ejecutivo a nivel regional podría ayudar a resolver estos problemas y, en consecuencia, exhortar la responsabilidad de los actores regionales. Esto supone una agenda de investigación que explore los mecanismos de 'accountability' horizontales y verticales. Esta perspectiva implica comprender el espacio regional como un espacio de deliberación en el escenario de una opinión pública regional. 
Debe destacarse que estas tres perspectivas no son incompatibles entre sí, sino que son tres maneras de comprender un mismo problema: la coordinación gubernamental. Por lo tanto, es posible desarrollar una agenda de investigación que las combine. Los desafíos del estudio del proceso de regionalización chileno implican el trabajo conjunto de múltiples disciplinas académicas como la administración pública, la ciencia política, los estudios urbanos, la sociología, la geografía, entre otras. La presente síntesis busca contribuir al diálogo, la reflexión y el trabajo multidisciplinario necesarios para el análisis de este proceso clave para el bienestar del país.

\section{Bibliografía}

Aguilar, L. F. 2009. Gobernanza y gestión pública. México D.F.: Fondo de Cultura Económica.

Aroca, P. y Atienza, M. 2010. "El mercado, sus agentes y la descentralización". Informe final. Estudio articulación y actores para la descentralización en tres regiones de Chile. Santiago: Programa Ciudadanía y Gestión Pública, Universidad de Los Lagos. 537-554. En línea, disponible en: http://www.innovacionciudadana.cl/portal/imagen/ File/descentralizacion/INFORME\%20FINAL.pdf (visitado el 14 de octubre de 2014).

Avendaño, O. 2008. "De la autonomía del mandato a la rendición de cuentas. Un alcance conceptual a los mecanismos de representación democrática". Revista de Sociología 22: 93-116.

Bache, I. y Flinders, M. 2004. Multi-level governance. Oxford: Oxford University Press.

Barozet, E. 2006. "Relecturas de la noción de clientelismo: Una forma diversificada de intermediación política y social". Debate 69: 77-101.

. 2005a. "La compra de los votos en Chile o cómo se coopta a los sectores populares". Contribuciones 133: 10-15.

2005b. "Los nuevos patrones del clientelismo en las urbes chilenas. Reflexión acerca del uso político de las organizaciones comunitarias en Santiago Centro e lquique". Introducción a la teoría y práctica del análisis de redes sociales (ARS). Espinoza, V. y Porras, J. I. (Eds.). Santiago: Universidad Bolivariana-Universidad de Santiago de Chile. 361-400.

Bitrán, E. 2004. "Sistema de innovación, consorcios tecnológicos y clúster dinámicos en Chile". En foco 20: 1-16. En línea, disponible en: http://www.expansiva. cl/media/en_foco/documentos/20092004100615.pdf (visitado el 23 de febrero de 2013).

Boisier, S. 2004. "Desarrollo territorial y descentralización. El desarrollo en el lugar y en las manos de la gente". Revista EURE 30 (90): 27-40. 2003. El desarrollo en su lugar. Santiago: Universidad Católica de Chile.

Centro de Estudios en Economía y Negocios. 2011. Octavo índice de competitividad de las regiones de Chile. En línea, disponible en: http://negocios.udd.cl/ ceen/estudios-y-publicaciones/icore/ (visitado el 27 de septiembre de 2012).

Comisión Asesora Presidencial para la Descentralización y el Desarrollo Regional. 2014. Propuesta de Política de Estado y Agenda para la Descentralización y el Desarrollo Territorial de Chile. Hacia un país desarrollado y justo. En línea, disponible en: http://descentralizacion.cl/?wpfb_dl=78 (visitado el 5 de diciembre de 2014).

De la Maza, G. 2012. "Espacio público y participación ciudadana en la gestión pública en Chile: Límites y posibilidades". Revista Polis 30. En línea, disponible en: http://polis.revues.org/2132 (visitado el 25 de mayo de 2013).

., Cunill, N. y Joignant, A. 2012. "La descentralización: Asunto de actores y su articulación". Nueva agenda de descentralización en Chile. Sentando más actores a la mesa. Joignant, A., De la Maza, G. y Cunill, N. (Eds.). Santiago: RIL Editores-Universidad de Los Lagos. 21-65.

2010. "Participación ciudadana en experiencias de desarrollo territorial: Contribuciones a la gobernanza democrática". Informe final. Estudio articulación y actores para la descentralización en tres regiones de Chile. Santiago: Programa Ciudadanía y Gestión Pública, Universidad de Los Lagos. 16-37. En línea, disponible en: http://www.innovacionciudadana.cl/portal/imagen/File/ descentralizacion/INFORME\%20FINAL.pdf (visitado el 14 
de octubre de 2014).

Durston, J. 2012. "Clientelismo político y actores populares en tres regiones de Chile". Nueva agenda de descentralización en Chile. Sentando más actores a la mesa. De la Maza, G., Cunill, N. y Joignant, A. (Eds.). Santiago: RIL Editores-Universidad de Los Lagos. 369-392.

et al. 2005. Comunidades campesinas, agencias públicas y clientelismos políticos en Chile. Santiago: LOM.

Espinoza, V. y Bustamante, C. 2015. "Redes políticas de decisión regional. ¿Quién tiene el poder en las regiones?". Seminario COES-CISPO: Territorios, redes y singularidades políticas. Santiago, Chile.

. y Rabi, V. 2013. "La gobernanza del desarrollo regional y la toma de decisiones para inversión pública regional en Chile". XXIX Congreso Asociación Latinoamericana de Sociología. Santiago, Chile. 2006. "Los nuevos agentes políticos locales: Revisión estructural de la tesis de Arturo Valenzuela. ¿Cómo se articulan el nivel de representación local con el nacional en la arena local y qué papel juegan el gobierno central y las políticas públicas?". Revista MAD 14: 151-164.

Felzensztein, C. y Olavarría, J. 2011. Innovación regional y clústeres: Lecciones para Chile y Latinoamérica. Santiago: Editorial Andrés Bello.

Fløysand, A., Barton, J. y Román, A. 2010. "La doble jerarquía del desarrollo económico y gobierno local en Chile: El caso de la salmonicultura y los municipios chilotes". Revista EURE 36 (108): 123-148.

Garcés, R. y Valdés, T. 2009. "Descentralización: Cuánto hemos avanzado en los últimos 20 años". Informe Instituto Libertad XX (216): 23-24.

González, R. 2010. "CORFO, fomento productivo, innovación y articulación de actores". Informe final. Estudio articulación y actores para la descentralización en tres regiones de Chile. Santiago: Programa Ciudadanía y Gestión Pública, Universidad de Los Lagos. 472-511. En línea, disponible en: http://www.innovacionciudadana. cl/portal/imagen/File/descentralizacion/INFORME\%20 FINAL.pdf (visitado el 14 de octubre de 2014).

Horst, B. 2009. "Fuentes de financiamiento para gobiernos subnacionales y descentralización". Un mejor Estado para Chile: Propuestas de modernización y reforma. Tomicic, V. y García, C. (Eds.). Santiago: Pontificia Universidad Católica de Chile-Consorcio para la Reforma del Estado. 207-240.

, Irarrázaval, I. y Pérez, E. 2009. “Institucionalidad subnacional y el problema de coordinación de competencias". Un mejor Estado para Chile: Propuestas de modernización y reforma. Tomicic, V. y García, C. (Eds.). Santiago: Pontificia Universidad Católica de Chile-
Consorcio para la Reforma del Estado. 175-205. 2008. "Gobiernos regionales, una verdad incómoda". Rompiendo cadenas del centralismo en Chile. Horst, B., Irarrázaval, I. y Del Río, F. (Eds.). Santiago: Libertad y Desarrollo-Pontificia Universidad Católica de Chile. 111-133.

Jabko, N. 2004. "The importance of being nice: An institutionalist analysis of French preferences on the future of Europe". Comparative European Politics 2: 282-301.

Klijn, E. 1998. "Policy network: An overview". Managing complex networks. Kickert, W. J. M. y Koppenjan, F. F. (Eds.). London: SAGE Publications.15-35.

Knoke, D. 1993. "Networks of elite structure and decision making". Sociological Methods Research 22 (1): 23-45.

y Pappi, F. U. 1991. "Organizational action sets in the United-States and German labor policy domains". American Sociological Review 56 (4): 509-523.

., Laumann, E. y Kim, Y. 1985. "An organizationa approach to state policy formation: A comparative study of energy and health domains". American Sociology Review 50 (1): 1-19.

Kollock, P. 1998. "Social dilemmas: The anatomy of cooperation". Annual Review of Sociology 24 (1): 183-214.

Larraín, H. 2008. "De la actual re-centralización a la descentralización en Chile". Rompiendo cadenas del centralismo en Chile. Horst, B., Irarrázaval, I. y Del Río, F. (Eds.). Santiago: Libertad y Desarrollo-Pontificia Universidad Católica de Chile. 131-151.

Lechner, N. 1997. "Tres formas de coordinación social". Revista CEPAL 61: 7-17.

Marcel, M. 2006. Acreditación y mejora continua de los procesos de gestión subnacional en Chile. En línea, disponible en: http://www.subdere.gov.cl/sites/default/ files/documentos/articles-77337_recurso_3.doc (visitado el 15 de junio de 2014).

Mardones, R. 2008. "Descentralización: Una definición y una evaluación de la agenda legislativa chilena (19902008)". Revista EURE 34 (102): 39-60.

2006. "Descentralización y transición en Chile". Revista de Ciencia Política 26 (1): 3-24.

2001. "Impacto económico de la descentralización en Chile: Evaluación actual y prospectiva. Más allá del bosque". Transformar el modelo de desarrollo. Muñoz, O. (Ed.). Santiago: FLACSO. 269-302.

Mascareño, A. 2010. "Coordinación social mediante políticas públicas: El caso chileno". Revista CEPAL 101: 111-126.

Montecinos, E. 2011. "Democracia participativa y presupuesto participativo en Chile: ¿Complemento 
- subordinación a las instituciones representativas locales?". Revista de Ciencia Política 31 (1): 63-89. 2006. "Descentralización y democracia en Chile: Análisis sobre la participación ciudadana en el presupuesto participativo y el plan de desarrollo comunal". Revista de Ciencia Política 26 (2): 191-208.

Montero, C. 2004. Formación y desarrollo de un clúster globalizado: El caso de la industria del salmón en Chile. Santiago: CEPAL.

y Morris, P. 1999. Estudio sobre el sistema de innovación regional. Programa de innovación tecnológica. Región de O’ Higgins: Ministerio de Economía.

Navarrete, B. e Higueras, V. 2014. "Chile desde la teoría secuencial de la descentralización, 1990-2010". Convergencia 66: 179-202.

2010. "Los problemas de articulación de intereses en las regiones de Chile (1989-2009)". Informe final. Estudio articulación y actores para la descentralización en tres regiones de Chile. Santiago: Programa Ciudadanía y Gestión Pública, Universidad de Los Lagos. 277-334. En línea, disponible en: http://www.innovacionciudadana. $\mathrm{cl} /$ portal/imagen/File/descentralizacion/INFORME\%20 FINAL.pdf (visitado el 14 de octubre de 2014).

Nickson, A. 2011. Where is local government going in Latin America? A comparative perspective. Visby: Swidish International Centre for Local Democracy. En línea, disponible en: http://www.icld.se/pdf/ICLD_wp6_ printerfriendly.pdf (visitado el 15 de junio de 2014).

O'Donnell, G. 1999. "Horizontal accountability in new democracies". Self-restraining state: Power and accountability in new democracies. Shedler, A., Diamond, L. y Plattner, M. (Eds.). Colorado-London: Lynne Rienner. 112-126.

Pawson, R. 2006. Evidence-based policy: A realist perspective. London: SAGE Publications. y Tilley, N. 1997. Realistic evaluation. London: SAGE Publications.

Pérez-Alemán, P. 2005. "CLUSTER formation, institutions and learning: The emergence of clusters and development in Chile". Industrial and Corporate Change 14 (4): 655-677.

Peruzzotti, E. y Smulovitz, C. 2002. Controlando la política. Buenos Aires: Temas.

Podolny, J. M. y Page, K. L. 1998. "Network forms of organization”. Annual Review of Sociology 24 (1): 57-76.

Porras, J. I. y Pino, R. 2010. "Concertación público y privada y redes de política pública”. Informe final. Estudio articulación y actores para la descentralización en tres regiones de Chile. Santiago: Programa Ciudadanía y Gestión Pública, Universidad de Los Lagos. 555-590. En línea, disponible en: http://www.innovacionciudadana. cl/portal/imagen/File/descentralizacion/INFORME\%20 FINAL.pdf (visitado el 14 de octubre de 2014).

Porter, M. 1998. "Clusters and the new economics of competition”. Harvard Business Review 76 (6): 77-90.

Rivas, G. 2012. La experiencia de CORFO y la transformación productiva de Chile. Evolución, aprendizaje y lecciones de desarrollo. Corporación Andina de Fomento-Banco de Desarrollo de América Latina. En línea, disponible en: http://publicaciones.caf.com/ media/17638/caf_libro_corfo_web.pdf (visitado el 15 de junio de 2014).

Rehren, A. 2002. "Clientelismo político, corrupción y reforma del estado en Chile". Reforma del Estado. Volumen II. Dirección pública y compras Públicas. Valdés, S. (Ed.). Santiago: CEP. 127-164.

Rufian, D. M. 2009. Políticas e instituciones para el desarrollo económico territorial. El caso de Chile. Santiago: ILPES.

Sartori, G. 2002. Elementos de teoría política. Madrid: Alianza Editorial.

Serrano, C. 2001. "Inversión pública y gestión regional. Nudos críticos". Descentralización: Nudos críticos. Raczynski, D. y Serrano, C. (Eds.). Santiago: CIEPLANAsesorías para el Desarrollo. 27-172.

Silva, I. 2005. "Desarrollo económico local y competitividad territorial en América Latina". Revista CEPAL 89: 81-100.

Smulovitz, C. 2002. “¿Cómo consigue imperar el imperio de la ley? Imposición de costos a través de mecanismos descentralizados". Política y Gobierno 9 (2): 297-324.

y Peruzzotti, E. 2000. "Social accountability: The other side of control". Journal of Democracy 11 (4): 147-158.

Tello, M. D. 2010. "Del desarrollo económico nacional al desarrollo local: Aspectos teóricos". Revista CEPAL 102: 51-67.

Valenzuela, A. 1977. Political brokers in Chile: Local government in a centralized polity. Durham, N.C.: Duke University Press.

Vázquez, A. 2004. "Desarrollo endógeno: Interacción de las fuerzas que gobiernan los procesos de crecimiento económico". En la frontera del desarrollo endógeno. Vergara, P. y Von Vaer, H. (Eds.). Temuco: Ediciones Universidad de La Frontera. 223-253.

Zeng, M. y Chen, X. 2003. "Achieving cooperation in multiparty alliances: A social dilemma approach to partnership management". The Academy of Management Review 28 (4): 587-605. 